\title{
Miliary Tuberculosis with Severe Pneumonia without Abnormal Chest Sounds in a Covid-19 Pandemic
}

\author{
Atana Uket Ewa ${ }^{1,3 *}$; Glory Ekpo Bassey ${ }^{1,3}$; Ubong Aniefiok Udoh ${ }^{2,4}$; Enobong Ufot Akpah ${ }^{3}$; Kingsley Chidi Anachuna ; Bassey \\ Ewa Ekeng * \\ ${ }^{1}$ Department of Paediatrics, University of Calabar, Calabar, Nigeria. \\ ${ }^{2}$ Departments of Medical Microbiology and Parasitology, University of Calabar, Nigeria. \\ ${ }^{3}$ Department of Paediatrics, University of Calabar Teaching Hospital, Calabar, Nigeria. \\ ${ }^{4}$ Departments of Medical Microbiology and Parasitology, University of Calabar Teaching Hospital, Calabar, Nigeria.
}

\section{*Corresponding Author(s): Bassey Ewa Ekeng ${ }^{1}$ \&} Atana Uket Ewa²

${ }^{1}$ Medical Microbiology and Parasitology University of Calabar Teaching Hospital Calabar.

Tel: +2348037361816; Email: basseyewaekeng@gmail.com ${ }^{2}$ Department of Paediatrics, University of Calabar, Calabar, Nigeria.

Tel: +23408182867676; Email: atanaewa@yahoo.com

Received: Aug 06, 2021

Accepted: Aug 27, 2021

Published Online: Aug 30, 2021

Journal: Journal of Tuberculosis

Publisher: MedDocs Publishers LLC

Online edition: http://meddocsonline.org/

Copyright: (C) Ekeng BE (2021). This Article is

distributed under the terms of Creative Commons

Attribution 4.0 International License

Keywords: Miliary TB; Chest Xray; Xpert MTB/Rif; Covid-19; Oxygen saturation.

\section{Abstract}

Background: Tuberculosis (TB) is still causing deaths in children in TB endemic countries. Majority (80\%) occur in the lungs, with $5 \%$ being miliary TB. It is an Interstitial Lung Disease (ILD) with similar clinical, physiological and radiological features to other ILD and affects mainly infants and children with a high mortality rate despite available treatment.

Case Presentation: A three-month old male presented during the COVID-19 pandemic with fever of 2 weeks, cough of one week, fast and difficult breathing of 6 days' duration. He received $B C G$ vaccine 16 days after birth despite being unknowingly exposed to the TB contact from birth. He was acutely ill looking, with severe pneumonia and SPO2 of 76\% in room air. Respiratory examination showed broncho vesicular breath sounds with no added sounds. A diagnosis of Severe pneumonia? Pneumocystis Jiroveci Pneumonia (PJP) and R/O COVID-19 was made. Subsequent reviews excluded PJP and COVID-19 and the final diagnosis was Disseminated (Miliary) TB. He was successfully treated with anti-TB medications for 12 months.

Conclusion: Miliary TB presents with common and uncommon manifestations that may be confusing, and so a high index of suspicion with a careful history, focused systemic examination, imaging and bacteriological studies are strongly recommended for its early diagnosis. 


\section{Introduction}

Tuberculosis (TB) is an old disease still causing deaths in children in developing countries and TB endemic regions [1-3] and yet childhood TB remains under-diagnosed and under-reported [2]. Majority (80\%) of TB in children occur in the lungs, out of which $5 \%$ are miliary TB [2]. The term miliary TB was formed in 1700 by John Jacob Manget from the word 'miliarius' which means "related to millet seed" and represents disseminated TB with miliary shadows on CXR [4]. It has been a diagnosis of infants and children since the pre-antibioctic era [5] with a high mortality rate despite available treatment [4]. It is rare in children who have had BCG vaccination as this is known to reduce its incidence [4]. The diagnosis is challenging $[4,5,6]$ because it is an Interstitial Lung Disease (ILD) with similar clinical, physiological and radiological features to other ILD [4]. It can affect any tissues but usually, highly vascularised ones like the lungs, liver, spleen, bone marrow and kidneys are commonly involved [1].

Generally, clinical manifestations range from Adult Respiratory Distress Syndrome (ARDS) to Pyrexia of Unknown Origin (PUO) $[6,7]$ and these include fever (100\%), fatigue (91\%) and miliary shadows on CXR (88\%) [7]. Frequent presentations include fever, loss of appetite, weight loss, crepitations, hepatomegaly, splenomegaly, neurologic manifestations like facial nerve palsy and tuberculous meningitis $[2,8,10]$, but less commonly, chills, night sweats [11], daily morning fevers, haemoptysis, productive cough $[4,8]$, granulomas/tuberculomas, $[4,10,12]$ non-specific [13] and atypical presentations do occur[4]. Hepatosplenomegaly and peripheral lymphadenopathy are more frequent in children than in adults [8] and tuberculous meningitis occurs in $20-40 \%$ of cases compared to adults' 15 $30 \%$ [8] The risk factors for acquiring miliary TB are HIV, childhood infections, malnutrition, chronic kidney disease, organ transplant, immunosuppression [8].

Initially, there's hyperinflation with no classical miliary tubercles in $50 \%$ of patients. But generally CXR findings are consistent and identify miliary shadows in $91 \%$ of cases of miliary TB [14] with a sensitivity of $59-69 \%$ and a specificity of $97-100 \%$ $[4,5,7,9,11,14,15]$ Such findings include millet sized tubercles $<2 \mathrm{~mm}$ with $10 \%$ having nodules $>3 \mathrm{~mm}$ which may be asymmetrical, mottled, coalesce or give a snowstorm pattern [4]. Treatment of Miliary TB is with anti-Tb drugs and use of steroids is controversial [4].

Miliary TB therefore presents with a wide variety of common and uncommon manifestations that may confuse even the most experienced Clinician [5], and hence this report aims to add to the available literature of knowledge.

\section{Case presentation}

A three-month old male presented in our facility with fever of 2 weeks, cough of one week and fast breathing of 6 days duration.

The fever was initially low grade, intermittent but became high grade and continuous. Cough was insidious in onset, dry, non-paroxysmal but distressing and worsened over time with associated fast and difficult breathing. There's history of contact with a 20 year old aunty who had TB and had been on treatment for 2 months before baby's presentation. Pregnancy was booked at a Primary Health Center (PHC) at 6 months' gestation and mother had regular attendance and was compliant on her routine drugs and vaccinations. Foetal ultrasound scan was normal and HIV screening was negative. The pregnancy was uneventful and carried to term with uneventful delivery and neonatal period. He was exclusively breastfed and immunized for age but noticed to have received BCG 16 days after birth despite being unknowingly exposed to the TB source contact right from birth. The source TB contact was diagnosed and started on AntiTB treatment one month after his birth. He's the second of two siblings with the senior, a 2 year old female. Mother is 23 year old Secondary School Certificate of Education (SSCE) holder and father is a $\mathbf{3 1}$ year old with SSCE. All live in a poorly ventilated 2 room apartment.

Physical examination revealed an acutely ill looking male baby, in respiratory distress with flaring alae nasi, intercostal and subcostal recessions, febrile with temperature $38.5^{\circ} \mathrm{C}$, respiratory rate-56/min, pulse rate- $154 / \mathrm{min}, \mathrm{SPO}_{2}-76 \%$ in room air, mildly pale, with a weight of $4.4 \mathrm{~kg}$, occipitofrontal circumference $-38 \mathrm{~cm}$, Length- $55 \mathrm{~cm}$ and weight for length 0 to -1 . Respiratory examination showed a symmetrical chest, resonant percussion notes, bronchovesicular breath sounds with no added sounds. The central nervous system examination was also normal. The abdomen was full, soft with tender hepatomegaly of $4 \mathrm{~cm}$ and a splenomegaly of $3 \mathrm{~cm}$. The working diagnosis was underweight malnutrition with severe pneumonia? Pneumocystis Jirovecii Pneumonia (PJP) and rule out COVID-19. Subsequent reviews excluded PJP and COVID-19 and the final diagnosis was Disseminated (Miliary) TB.

HIV Screening for baby and mother were both negative. SARS COV-2 PCR was negative. CXR had widespread miliary nodules in all the segments and lobes of both lungs with attempts at cavitation in both upper lobes (Figure 1). Full blood count showed a total WBC of $18.3 \times 10^{9} / \mathrm{L}, \mathrm{PCV}-28 \%$, L-36\%, N-64\% with hypochromasia and microcytosis. Early morning gastric aspiration for Xpert MTB/Rif detected MTB sensitive to Rifampicin.

Patient remained acutely ill, dyspnoeic and tachypnoeic with RR-56/min, having severe pneumonia on intranasal oxygen with $\mathrm{SPO}_{2}$ ranging from $70-85 \%$ in room air and $92-98 \%$ on oxygen, yet with bronchovesicular sounds, good air entry bilaterally and no added sounds. Three days into admission and second day after starting anti-TB drugs (Rifampicin, Isoniazid, Pyrazinamide and Ethambutol for 2 months, followed by Rifampicin and Isoniazid for 10 months), he developed generalized tonic clonic seizures with temp $38.3^{\circ} \mathrm{C}$ aborted with intravenous diazepam. The seizures recurred after 24 hours with 4 episodes in all and a suspicion of tuberculous meningitis was entertained in addition. Phenobarbitone and prednisolone were then commenced after aborting the seizures with diazepam. Lumbar puncture was planned to rule out tuberculous meningitis but he remained very ill.

By the $4^{\text {th }}$ day of anti-TB treatment, the temperature had stabilized for $48 \mathrm{hrs}$, he was tolerating expressed breastmilk and $\mathrm{SPO}_{2}$ in room air improved to $93-100 \%$ and oxygen therapy was subsequently discontinued. He was much improved but still ill-looking, in mild respiratory distress with RR-49/min and the chest examination still remained normal with resonant percussion notes, bronchovesicular and no added sounds. His improvement on anti-TB medications was so sustained that on the $9^{\text {th }}$ day of admission, he was discharged home, to be followed up in the clinic 2 weeks later. He defaulted from the clinic visit for logistic reasons but was however collecting and adhering to anti-Tb drugs as prescribed and presented after 2 months of intensive phase at the age of 5 months. The mother complained of intermittent fever and inability to hold up the neck. 
He had attained neck control before the onset of the illness at 3 months of age and was yet to regained it. Weight gain was also slow with only $400 \mathrm{~g}$ added in 2 months since discharge. Physical examination showed head lag but normal tone, mild pallor, RR$52 / \mathrm{min}$, clear chest with normal cardiovascular system and gastrointestinal tract. Diagnosis remained disseminated TB involving miliary and tuberculous meningitis in an underweight child. Nutritional counselling was reinforced, continuation phase of treatment commenced and steroid therapy with prednisolone which had been interrupted was re-commenced for 4-6 weeks. He has been regular on his Anti-TB medications, seen again three times in the outpatient clinic and had shown sustained improvement. By 9 months of age and 7th month of Anti-TB

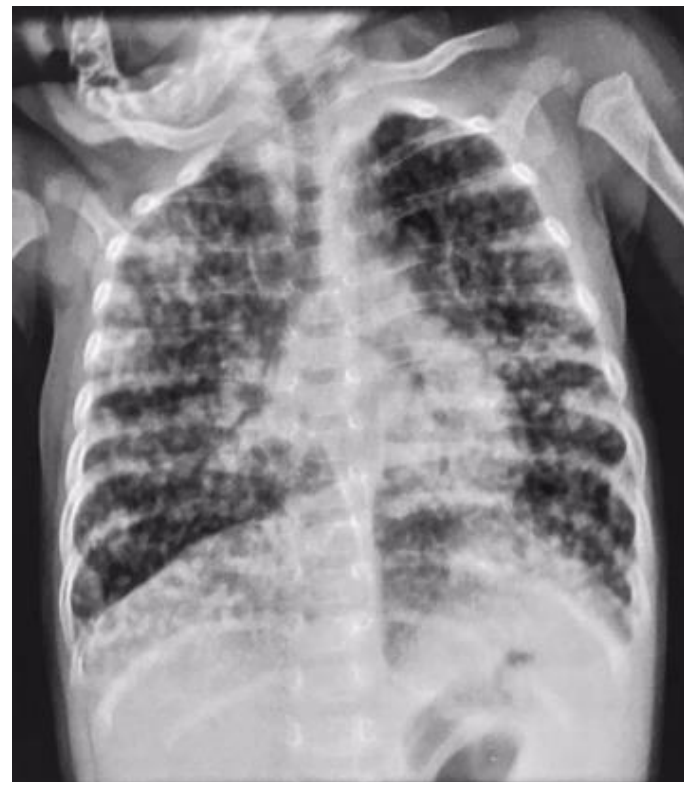

Figure 1: CXR of the 3 month's old male child on admission. CXR showed widespread miliary nodules in all the segments and lobes of both lungs with attempts at cavitation in both upper lobes.

\section{Discussion}

Our case presented with fever, cough, difficult and fast breathing with clinical features of severe hypoxia recording very low oxygen saturation in room air. This severe pneumonia with desaturation in the present pandemic was so confusing that our closest differentials were COVID-19, as reported by studies $[16,17]$ followed by PJP. Both were excluded by their negative test results and outcome.

He was acutely ill looking, in respiratory distress, febrile, mildly pale, with a clinically clear chest. Even though studies have shown the non-specific symptomatology and presentation of miliary TB $[4,6,14]$, none have reported persistently clear chest despite such severe pneumonia associated with desaturation as found in our patient.

RDS as found in our patient is a known complication of miliary TB and has been closely associated with tuberculous meningitis $[2,18]$. Again, known risk factors for developing military TB are young age, lack of BCG, malnutrition and contact with Tb case [18], all of which were found in our patient. He did not receive $B C G$ until 16 days after birth which exposed him to the TB bacilli before the BCG vaccine. Miliary TB is rare in children who have had BCG vaccination as this is known to reduce its incidence $[4,8]$.

A tender hepatosplenomegaly as found in our case was a common finding reported in several studies $[1,2,9]$. treatment, he had gained weight up to $6.8 \mathrm{~kg}$, regained neck control at 7-8 months and had discontinued prednisolone again after 3 weeks. Physical examination was satisfactory with no respiratory distress and a clear chest, diagnosis remained disseminated TB with Miliary TB and probable tuberculous meningitis responding to treatment. A repeat CXR showed interval improvement with hyperinflation, persisting air bronchogram and a residual well circumscribed opacity in the lower lung lobe (Figure 2). His last visit was at 14 months of age and $11^{\text {th }}$ month of Anti-TB treatment with sustained and satisfactory improvement. He continues to be followed up in the Childrens' outpatient department, while Anti-TB treatment also continues for a total of 12 months.

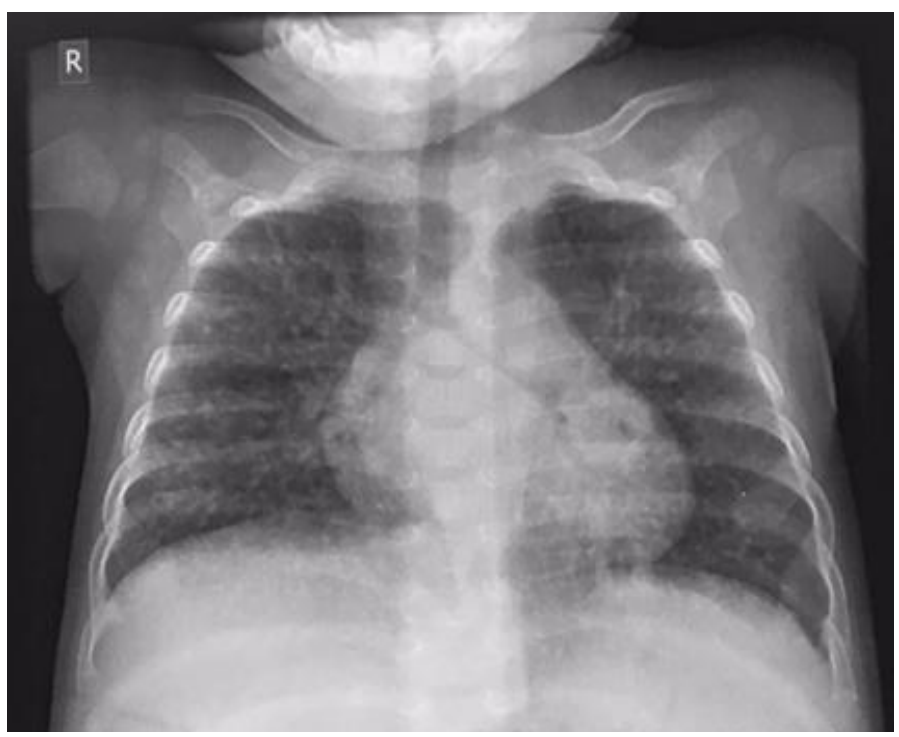

Figure 2: Repeat CXR of same patient $7^{\text {th }}$ month on Anti-TB treatment.

A repeat CXR showed interval improvement with hyperinflation, persisting air bronchogram and a residual well circumscribed opacity in the lower lung lobes.

Again, being a male child is supported by studies showing that males are more frequently affected by miliary TB in studied childhood and adult series [4].

Our patient developed episodes of convulsions. Studies have reported seizures in military Tb either due to associated hypoxia or complicated by tuberculous meningitis [18].

The uniqueness of the case is the persistently clear chest findings with the total absence of added sounds in spite of the severe illness and hypoxia. Other studies have documented crepitations, among other findings $[9,19]$.

CXR findings had widespread miliary nodules as seen in several studies $[7,14,19]$. The presence of residual opacities in the repeat CXR done at 7 months of treatment buttresses arguments to support extension of anti-TB treatment beyond 6 months for miliary TB which is what is currently being practiced in Nigeria [20-22].

The diagnosis of miliary Tb could be very challenging in resource poor settings, but as much as its possible every effort should be made to support CXR and bacteriological investigations in these areas for early diagnosis and prompt treatment $[5,19]$. 


\section{Conclusion}

Miliary TB presents with a wide range of common and uncommon manifestations that may confuse even the most experienced Clinician, and so a high index of suspicion with a careful history, focused systematic physical examination, imaging and bacteriological studies are strongly recommended for its early diagnosis. Prompt treatment with standard Anti-TB drugs is lifesaving.

\section{Ethical statement}

The study was conducted according to the principles of the World Medical Association Declaration of Helsinki and written informed consent was obtained from the parent for publication of this case report.

\section{Acknowledgement}

The authors wish to acknowledge both parents of the patient for their cooperation, especially during the critical phase of the illness and also for their understanding and perseverance throughout the treatment period for a successful outcome.

\section{Conflict of interest: None}

\section{Source of funding: None}

\section{Authors' contribution}

AUE-Conceptualization, collection of data, Resources, Writing-Original Draft Preparation, and Supervision, GEB-Resources, collection of data, Writing-Review \& Editing, UAU-Investigation, Resources, Writing-Review \& Editing, EUA-Resources, collection of data, Writing-Review \& Editing, KAC-Resources, collection of data, Writing-Review \& Editing, BEE-Investigation, Resources, Writing-Review \& Editing. All authors agreed to the final version of submitted manuscript.

\section{References}

1. Moule MG, Cirillo JD. Mycobacterium tuberculosis Dissemination Plays a Critical Role in Pathogenesis. Front. Cell. Infect. Microbiol. 2020; 10: 65.

2. Cruz AT, Starke JR. Pediatric Tuberculosis. Pediatrics in Review 2010; 31:13-25.

3. Marais BJ, Schaaf HS. Tuberculosis in Children. Cold Spring Harb Perspect Med 2014; 4: a017855.

4. Sharma SK, Mohan A. Miliary tuberculosis. Microbiol Spectrum 2017; 5: TNMI7-0013-2016.

5. Tanrıkulu C, Gürkan F, Dağı CE, Gözü A, Süner A. A comparative review of Pediatric and adult patients with miliary tuberculosis. Eur J Gen Med. 2007; 4: 67-72.

6. Sharma SK, Mohan A, Sharma A. Challenges in the diagnosis \& treatment of miliary tuberculosis. Indian J Med Res. 2012; 135: 703-730.
7. Mert A, Arslan F, Kuyucu T, Koç EN, Yılmaz M, et. al. Miliary tuberculosis Epidemiological and clinical analysis of large-case series from moderate to low tuberculosis endemic Country. Medicine. 2017; 96: e5875.

8. Sharma SK, Mohan A, Sharma A. Miliary tuberculosis: A new look at an old foe. Journal of Clinical Tuberculosis and Other Mycobacterial Diseases. 2016; 3: 13-27.

9. Gurkan F, Bosnak M, Dikici B, Bosnak V, Yaramis A, et.al. Miliary Tuberculosis in Children: A Clinical Review. Scan J Infect Dis. 1998; 30: 359-362.

10. St. Cyr G, Starke, Jeffrey R. Multiple Cranial Tuberculomas Without Meningitis in Two Infants With Miliary Tuberculosis. The Pediatric Infectious Disease Journal 2019; 38: e337-e339.

11. Kwong JS, Carignan S, Kang EY, Müller NL, FitzGerald JM. Miliary tuberculosis. Diagnostic accuracy of chest radiography. Chest. 1996; 110: 339-342.

12. Brian NK, Thwaites RG. The mechanisms and consequences of the extra-pulmonary dissemination of Mycobacterium tuberculosis. Tuberculosis 2010; 6: 361-366.

13. Lee JS, Lim CH, Kim E, Lim H, Lee Y, Choung JT, et.al. Congenital miliary tuberculosis in an 18-day-old boy. Korean J Pediatr. 2016; 59: S64-S67.

14. Gregory H, Terry C, Maurice K. Miliary tuberculosis in children: a review of 94 cases. The Pediatric Infectious Disease Journal. 1991; 10: 832-836.

15. Schaaf HS, Gie RP, Beyers N, Smuts N, Donald PR. Tuberculosis in infants less than 3 months of age. Arch Dis Child. 1993; 69: 371374.

16. Patnaik S, Beher JR, Nayak MK, Pradhan JB, Bikash Ranjan Sahu BR, et al. COVID-19 in Children: Present and Future Perspective, An Interim Review. India J Child Sci. 2020; 10: e53-e62.

17. Saunders MJ, Evans CA. COVID-19, tuberculosis, and poverty: Preventing a perfect storm. Eur Respir J 2020.

18. Clarissa CM, Budi U, Retno AS. Risk factors for miliary tuberculosis in children. Paediatr Indones. 2017; 57: 63-66.

19. Khan FY. Review of literature on disseminated tuberculosis with emphasis on the focused diagnostic workup. J Family Community Med. 2019; 26: 83-91.

20. Federal Ministry of Health. National Tuberculosis, Leprosy and Buruli Ulcer Management and Control Guidelines. National Tuberculosis and Leprosy Control Programme, Department of Public Health. 2015; 6: 52-67.

21. FMOH-NTBLCP/WHO/USAID/TB CARE 1. Desk Guide for the Diagnosis and Management of TB in Children in Nigeria. 2016; 2. FNWHOUTBC 1.

22. Federal Ministry of Health. Orientation manual for health care providers on the new Paediatric anti - tuberculous drug formulation. Department of Public Health, National Tuberculosis and Leprosy Control Programme. 2017. 